\title{
Matter Localization on Brane-Worlds Generated by Deformed Defects
}

\author{
Alex E. Bernardini' ${ }^{1}$ and Roldão da Rocha ${ }^{2}$ \\ ${ }^{1}$ Departamento de Física, Universidade Federal de São Carlos, P.O. Box 676, 13565-905 São Carlos, SP, Brazil \\ ${ }^{2}$ Centro de Matemática, Computação e Cognição, Universidade Federal do ABC (UFABC), 09210-580 Santo André, SP, Brazil
}

Correspondence should be addressed to Roldão da Rocha; roldao.rocha@ufabc.edu.br

Received 14 March 2016; Revised 21 April 2016; Accepted 16 June 2016

Academic Editor: Barun Majumder

Copyright ( 2016 A. E. Bernardini and R. da Rocha. This is an open access article distributed under the Creative Commons Attribution License, which permits unrestricted use, distribution, and reproduction in any medium, provided the original work is properly cited. The publication of this article was funded by SCOAP ${ }^{3}$.

\begin{abstract}
Localization and mass spectrum of bosonic and fermionic matter fields of some novel families of asymmetric thick brane configurations generated by deformed defects are investigated. The localization profiles of spin 0 , spin $1 / 2$, and spin 1 bulk fields are identified for novel matter field potentials supported by thick branes with internal structures. The condition for localization is constrained by the brane thickness of each model such that thickest branes strongly induce matter localization. The bulk mass terms for both fermion and boson fields are included in the global action as to produce some imprints on mass-independent potentials of the Kaluza-Klein modes associated with the corresponding Schrödinger equations. In particular, for spin 1/2 fermions, a complete analytical profile of localization is obtained for the four classes of superpotentials here discussed. Regarding the localization of fermion fields, our overall conclusion indicates that thick branes produce a left-right asymmetric chiral localization of spin $1 / 2$ particles.
\end{abstract}

\section{Introduction}

The brane-world model is a prominent paradigm that has been addressed to solve several questions in physics. Within this framework, brane-worlds are required to render a consistent $4 D$ physics of our Universe, at least up to certain sensible limits [1]. In the brane-world scenario all kinds of matter fields should be localized on the brane. In the RS brane-world model [2], the brane is generated by a scalar field coupled to gravity $[3,4]$, in a particular scenario which may be interpreted as the thin brane limit of thick brane scenarios. Generically, a prominent test that thick brane-world models must pass, to be physically consistent, regards their stability, with respect to tensor, vector, and scalar fluctuations of the background fields that generate the field configurations, namely, the thick brane itself. At least the zero modes of Standard Model matter fields were shown to be localized on several brane-world models [5-10], suggesting that such kind of models is physically viable in high energy physics. Several alternative scenarios, including Gauss-Bonnet terms, $f(R)$ gravity, tachyonic potentials, cyclic defects, and Bloch branes, have been further studied [11-15], and analogous scenarios in an expanding Universe have been approached [16, 17]. The curvature nature of the brane-world, namely, to be a de Sitter, Minkowski, or anti-de Sitter one, is in general obtained $a$ posteriori, by solving the $5 \mathrm{D}$ Einstein field equations. In fact, the bulk and the brane cosmological constants depend upon the brane and the bulk gravitational field content, governed by curvature, and must obey the intrinsic fine-tuning, in the Randall-Sundrum-like models limit.

The analytical study of stability can be uncontrollably intricate, due to the involved structure of the scalar field coupled to gravity. To circumvent the complicated and not analytical approaches, linearized formulations have been commonly worked out. In this context, supported by the stability of deformed defect generated brane-world models, scalar, vector, and tensor perturbations are investigated throughout this work.

Localization aspects of various matter fields with spin 0 , spin $1 / 2$, and spin 1 on analytical thick brane-world models are indeed a main concern in deriving brane-world models, since they must describe our physical $4 D$ world. The localization 
of the spin $1 / 2$ fermions deserves a special attention, since there is no scalar field to couple with in this model, in contrast to thick branes generated by deforming defect mechanisms [18]. Otherwise, Kalb-Ramond fields, although already investigated [19], will not be the main aim here. The spin $1 / 2$ issue has been previously studied in some other contexts [20], including further coupling of more scalar fields in the action [21] and asymmetric brane-worlds generated by a plenty of scalar field potentials [8, 22-25]. In particular, asymmetric Bloch branes in the context of the hierarchy problem have been addressed in [13].

Our aim is to investigate the localization of bulk matter and gauge fields on the brane, in the context where the massindependent potentials of the corresponding Schrödingerlike equations, regarding the $1 D$ quantum mechanical analogue problem, can be suitably acquired from a warped metric. In particular, for a bulk mass proportional to the fermion mass term enclosed by the global action, the possibility of trapping spin $1 / 2$ fermions on asymmetric branes is discussed and quantified.

To accomplish this aim, this paper is organized as follows. In Section 2, a brief review of brane-world scenarios supported by an effective action driven by a (dark sector) scalar field is presented. Warp factors and the corresponding internal brane structure are described for four different analytical models. In Section 3, the left-right-chiral asymmetric aspects of matter localization for spin 1/2 fermion fields on thick branes are investigated. Extensions to scalar boson and vector boson fields are obtained in Sections 4 and 5, respectively. Final conclusions are drawn in Section 6.

\section{Brane-World Preliminaries and Some Analytical Models}

Let one start considering a $5 D$ space-time warped into $4 D$. The most general $5 D$ metric compatible with a brane-world spatially flat cosmological background has the form given by

$$
d s^{2}=g_{M N} d x^{M} d x^{N}=e^{2 A(y)} g_{\mu \nu}\left(x^{\alpha}\right) d x^{\mu} d x^{\nu}+d y^{2},
$$

where $e^{2 A(y)}$ denotes the warp factor, and the signature $(-++++)$ is employed, with $M, N=0,1,2,3,5 . g_{\mu \nu}$ stands for the components of the $4 D$ metric tensor $(\mu, \nu=0,1,2,3)$. One can identify $y \equiv x_{4}$ as the infinite extra dimension coordinate (which runs from $-\infty$ to $\infty$ ) and notice that the normal to surfaces of constant $y$ is orthogonal to the brane, into the bulk (brane tension terms have been suppressed/ absorbed by the metric (c.f. Equations (24) and (25) from [10] for real scalar field Lagrangians in the context of thick brane solutions)).

The brane-world scenario examined here is set up by an effective action, driven by a (dark sector) scalar field, $\zeta$, coupled to $5 D$ gravity, given by

$$
\begin{aligned}
S_{\text {eff }} & =-\int d x^{5} \sqrt{\operatorname{det} g_{M N}}\left[\frac{1}{4}\left(\kappa_{5}^{-2} R-2 \Lambda_{5}\right)\right. \\
& \left.+\frac{1}{2} g_{M N} \partial^{M} \zeta \partial^{N} \zeta-V(\zeta)\right]
\end{aligned}
$$

where $R$ is the $5 D$ scalar curvature, $R_{N Q}=g^{B M} R_{B N Q M}$ is the Ricci tensor, and $\kappa_{5}=\left(8 \pi G_{5}\right)^{1 / 2}$ denotes the $5 D$ gravitational coupling constant, hereon set to be equal to unity, where $G_{5}$ is the $5 D$ Newton constant. The Einstein equations read

$$
R_{M N}-\frac{1}{2} R g_{M N}=-\Lambda_{5} g_{M N}+\kappa_{5}^{2} T_{M N}^{\zeta}
$$

where $T_{M N}^{\zeta}$ denotes the energy-momentum tensor corresponding to the matter Lagrangian, regarding the matter field $\zeta$. After solving the $5 D$ Einstein field equations, the bulk cosmological constant turns out, in general, to be positive or negative, thus realising a de Sitter or anti-de Sitter braneworld, respectively, generated by curvature. It realises and emulates the interplay involving the $4 D$ and $5 D$ cosmological constants. Some further possibilities are devised, for example, in $[14,26]$; however it is worth mentioning that an additional scalar field can be still added in the action, whose isotropisation will precisely define the nature of the braneworld. This latter case is however beyond the scope of our analysis. Obviously, whatever the possibility to be considered, the thin brane limit must obey the fine-tuning relation [27] $\Lambda_{4}=\left(\kappa_{5}^{2} / 2\right)\left((1 / 6) \kappa_{5}^{2} \sigma^{2}+\Lambda_{5}\right)$, among the effective $4 D$ and $5 D$ cosmological constants and the brane tension $\sigma$ as well.

Considering the real scalar field action, (2), one can compute the stress-energy tensor

$$
T_{M N}^{\zeta}=\partial_{M} \zeta \partial_{N} \zeta+g_{M N} V(\zeta)-\frac{1}{2} g_{M N} g^{A B} \partial_{A} \zeta \partial_{B} \zeta
$$

which, supposing that both the scalar field and the warp factor dynamics depend only upon the extra coordinate, $y$, leads to an explicit dependence of the energy density in terms of the field, $\zeta$, and of its first derivative, $d \zeta / d y$, as

$$
T_{00}^{\zeta}(y)=\left[\frac{1}{2}\left(\frac{d \zeta}{d y}\right)^{2}+V(\zeta)\right] e^{2 A(y)} .
$$

With the same constraints on $\zeta$ about the dependence on $y$, the equations of motion currently known from $[3,4]$, which arise from the above action, are

$$
\frac{d^{2} \zeta}{d y^{2}}+4 \frac{d A}{d y} \frac{d \zeta}{d y}-\frac{d}{d \zeta} V(\zeta)=0
$$

through a variational principle relative to the scalar field, $\zeta$, and

$$
\frac{3}{2} \frac{d^{2} A}{d y^{2}}=-\left(\frac{d \zeta}{d y}\right)^{2}
$$

through a variational principle relative to the metric, or equivalently to $A$, manipulated to result into

$$
3\left(\frac{d A}{d y}\right)^{2}=\frac{1}{2}\left(\frac{d \zeta}{d y}\right)^{2}-V(\zeta),
$$

after an integration over $y$.

For the scalar field potential written in terms of a superpotential, $w$, as

$$
V(\zeta)=\frac{1}{8}\left(\frac{d w}{d \zeta}\right)^{2}-\frac{1}{3} w^{2}
$$


the above equations are mapped into first-order equations [3, 4] as

$$
\begin{aligned}
& \frac{d \zeta}{d y}=\frac{1}{2} \frac{d w}{d \zeta} \\
& \frac{d A}{d y}=-\frac{1}{3} w
\end{aligned}
$$

for which the solutions can be found straightforwardly through immediate integrations [3] (see also [10] and references therein). The energy density follows from (9) as

$$
T_{00}^{\zeta}(y)=\left[\frac{1}{4}\left(\frac{d w}{d \zeta}\right)^{2}-\frac{1}{3} w^{2}\right] e^{2 A(y)}
$$

The analysis of localization aspects of brane-world scenarios will be constrained by some known examples, I, II, III, and $\mathrm{IV}$, for which the warp factor, $A(y)$, and the energy density, $T_{00}(y)$, can be analytically computed. Model I is supported by a sine-Gordon-like superpotential given by

$$
w^{\mathrm{I}}(\zeta)=\frac{2}{\sqrt{2} a} \sin \left(\sqrt{\frac{2}{3}} \zeta\right)
$$

which reproduces the results from [4]. Model II corresponds to a deformed $\lambda \zeta^{4}$ theory with the superpotential given by

$$
w^{\mathrm{II}}(\zeta)=\frac{3 \sqrt{3}}{a}\left(1-\frac{\zeta^{2}}{9}\right)^{3 / 2}
$$

Models III and IV are deformed topological solutions from [28] supported by superpotentials like

$$
\begin{aligned}
& w^{\mathrm{III}}(\zeta)=\frac{2}{a} \arctan [\sinh (\zeta)] \\
& w^{\mathrm{IV}}(\zeta) \\
& =\frac{1}{4 a}\left[\zeta\left(5-2 \zeta^{2}\right) \sqrt{1-\zeta^{2}}+3 \arctan \left(\frac{\zeta}{\sqrt{1-\zeta^{2}}}\right)\right],
\end{aligned}
$$

where the parameter $a$ fixes the thickness of the brane described by the warp factor, $e^{2 A(y)}$. Besides exhibiting analytically manipulable profiles, the above superpotentials have already been discussed in the context of thick brane localization $[4,5,10]$. Models I and II are, respectively, motivated by sine-Gordon and $\lambda \zeta^{4}$ theories, and models III and IV are obtained (also analytically) from deformed versions of the $\lambda \zeta^{4}$ model [12]. In particular, models III and IV can also be mapped onto tachyonic Lagrangian versions of scalar field brane models $[6,10,28]$.
From the above superpotentials, the respective solutions for $\zeta(y)$ are set as

$$
\begin{aligned}
\zeta^{\mathrm{I}}(y) & =\sqrt{6} \arctan \left[\tanh \left(\frac{y}{2 \sqrt{2} a}\right)\right], \\
\zeta^{\mathrm{II}}(y) & =3 \operatorname{sech}\left(\frac{\sqrt{3} y}{2 a}\right), \\
\zeta^{\mathrm{III}}(y) & =\operatorname{arcsinh}\left(\frac{y}{a}\right) \\
\zeta^{\mathrm{IV}}(y) & =\frac{y}{\sqrt{a^{2}+y^{2}}}
\end{aligned}
$$

where one has suppressed any additional (irrelevant) constant of integration for convenience, and one has just considered the positive solutions (in (16) there could be explicit constant of integration that amounts to letting $y \mapsto y+C$, corresponding to the position of the brane in the extra dimension, for which one has set $C=0$ ).

The obtained expressions for the warp factor as resulting from (11) are, respectively, given by

$$
\begin{aligned}
& A^{\mathrm{I}}(y)=-\ln \left[\cosh \left(\frac{y}{\sqrt{2} a}\right)\right], \\
& A^{\mathrm{II}}(y)=\tanh \left(\frac{\sqrt{3} y}{2 a}\right)^{2}-2 \ln \left[\cosh \left(\frac{\sqrt{3} y}{2 a}\right)\right], \\
& A^{\mathrm{III}}(y)=\frac{1}{3}\left[\ln \left(1+\frac{y^{2}}{a^{2}}\right)-2 \frac{y}{a} \arctan \left(\frac{y}{a}\right)\right], \\
& A^{\mathrm{IV}}(y)=-\frac{1}{12}\left[\frac{y^{2}}{a^{2}+y^{2}}+3 \frac{y}{a} \arctan \left(\frac{y}{a}\right)\right],
\end{aligned}
$$

where integration constants are introduced as to set a normalization criterion for which $A(0)=0$.

The solutions for $A^{\mathrm{I}}$ and $A^{\mathrm{II}}$ are depicted in Figure 1. The corresponding localized energy densities computed through (12) are, respectively, given by

$$
\begin{aligned}
& T_{00}^{\mathrm{I}}(y)=\frac{3}{4 a^{2}} \operatorname{sech}\left(\frac{y}{\sqrt{2} a}\right)^{2}\left[\operatorname{sech}\left(\frac{y}{\sqrt{2} a}\right)^{2}\right. \\
& \left.-2 \tanh \left(\frac{y}{\sqrt{2} a}\right)^{2}\right], \\
& T_{00}^{\mathrm{II}}(y)=\frac{9}{8 a^{2}} \operatorname{sech}\left(\frac{\sqrt{3} y}{2 a}\right)^{8} \tanh \left(\frac{\sqrt{3} y}{2 a}\right)^{2} \\
& \cdot\left[7 \cosh \left(\frac{\sqrt{3} y}{a}\right)-\cosh \left(\frac{2 \sqrt{3} y}{a}\right)\right] \\
& \cdot e^{2 \tanh (\sqrt{3} y / 2 a)^{2}},
\end{aligned}
$$




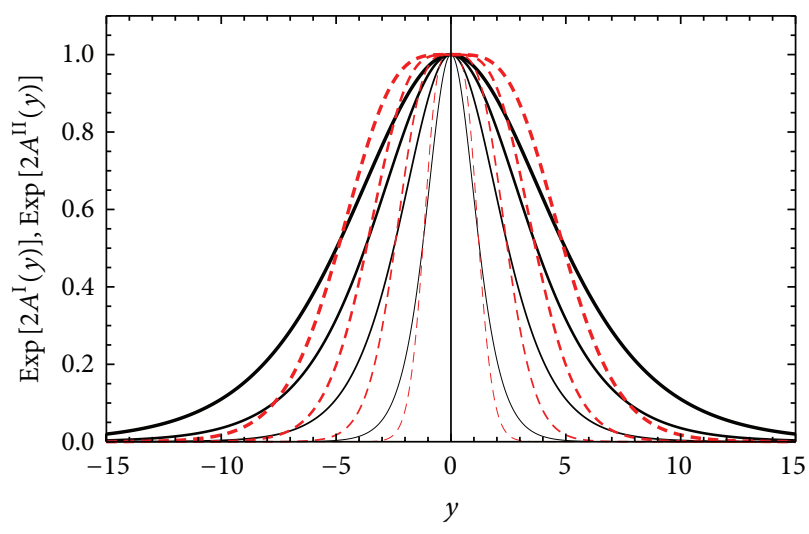

(a)

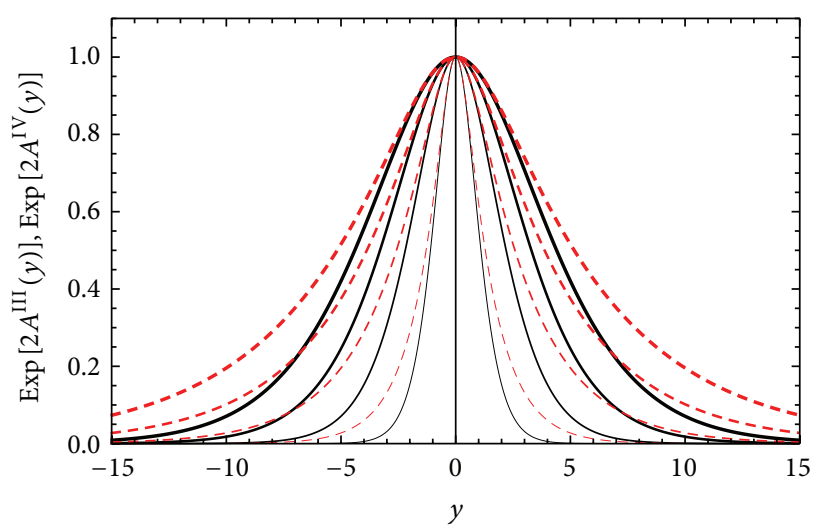

(b)

FIGURE 1: (a) Warp factors, $e^{2 A(y)}$ for models I (solid (black) lines) and II (dashed (red) lines). (b) Warp factors, $e^{2 A(y)}$ for models III (solid (black) lines) and IV (dashed (red) lines). One has considered integer values of the brane width parameter $a$, running from 1 (thinnest line) to 4 (thickest line), corresponding to an increasing thickness.

$$
\begin{aligned}
& T_{00}^{\mathrm{III}}(y)=\frac{1}{a^{2}}\left(1+\frac{y^{2}}{a^{2}}\right)^{-1 / 3}(1 \\
&-\left.\frac{4}{3}\left(1+\frac{a^{2}}{y^{2}}\right) \arctan \left(\frac{y}{a}\right)^{2}\right) e^{(4 y / 3 a) \arctan (y / a)}, \\
& T_{00}^{\mathrm{IV}}(y)=\left(\frac{a^{4}}{\left(a^{2}+y^{2}\right)^{3}}\right. \\
&\left.-\frac{5 a^{3} y+3 a y^{3}+3\left(a^{2}+y^{2}\right)^{2} \arctan (y / a)^{2}}{48 a^{2}\left(a^{2}+y^{2}\right)^{4}}\right) \\
& \cdot e^{-(y / 2 a)\left(\left(a y / 3\left(a^{2}+y^{2}\right)\right)+\arctan (y / a)\right)} .
\end{aligned}
$$

The brane scenarios for models from I to IV are depicted in Figure 1 for the warp factors and in Figure 2 for the energy densities, from which one can observe that models from I to IV give rise to thick branes, most of them with no internal structures. In fact, only the potential that controls the scalar field from model II allows the emergence of thick branes that host internal structures in the form of a layer of a novel phase enclosed by two separate interfaces, inside which the energy density of the matter field gets more concentrated. It is related to the extension/localization of the warp factor; namely, when the profiles depicted in Figure 1 approach a plateau form in the region inside the brane, the corresponding internal structure is observed through its energy profile.

The appearance of negative energy densities in the plots for $T_{00}$ may be related to a predominance of the scalar field potential over the kinetic-like term related to the coordinate $y$. Speculatively, it indicates that the vacuum minimal energy can be adjusted by the inclusion of some additional term, eventually related to the cosmological constant.

The localization of bulk matter fields on thick branes generated by each one of these models will be identified in the following sections. Spin 0 , spin $1 / 2$, and spin 1 fields will evolve coupled to gravity and, as usual, the bulk matter field contribution to the bulk energy will be neglected. It means that the obtained solutions hold in the presence of the bulk matter, without disturbing the bulk geometry.

\section{Asymmetric Left-Right Matter Localization for Spin 1/2 Fermion Fields}

To investigate the localization of bulk matter on the brane, one first considers that fermion localization on brane-worlds is usually accomplished when the $5 D$ Dirac algebra is realised by the objects $\Gamma^{M}=e_{\frac{M}{M}} \Gamma^{\bar{M}}$, where $e_{\bar{M}}^{M}$ denotes the fünfbein, $\Gamma^{M}$ satisfy the Clifford relation $\left\{\Gamma^{M}, \Gamma^{N}\right\}=2 g^{M N}$, and $\Gamma^{\bar{M}}$ are the gamma matrices in the $5 D$ flat space-time. Hereupon $\bar{M}, \bar{N}, \ldots=0,1,2,3,5$ and $\bar{\mu}, \bar{\nu}, \ldots=0,1,2,3$ denote the $5 D$ and $4 D$ local Lorentz indexes, respectively. The fünfbein is provided by $e_{M}^{\bar{M}}=\left\{e^{A} \widetilde{e}_{\mu}^{\bar{v}}, e^{A}\right\}$, where $\Gamma^{M}=e^{-A}\left(\gamma^{\mu}, \gamma^{5}\right)$, and $\gamma^{\mu}=\tilde{e}_{\bar{\gamma}}^{\mu} \gamma^{\bar{\nu}}$ and $\gamma^{5}$ are, respectively, the $4 D$ gamma matrices and the $4 D$ volume element, respectively. The Dirac action for a spin $1 / 2$ fermion with a mass term can be expressed as $[20,25]$

$$
\begin{aligned}
& S_{1 / 2} \\
& \quad=\int d^{5} x \sqrt{-g}\left[\bar{\Psi} \Gamma^{M}\left(\partial_{M}+\omega_{M}\right) \Psi-M F(z) \bar{\Psi} \Psi\right] .
\end{aligned}
$$

Here $\omega_{R}=(1 / 4) \omega_{R}^{\overline{R S}} \Gamma_{\bar{R}} \Gamma_{\bar{S}}$ is the spin connection, where

$$
\omega_{R}^{\bar{R} \bar{S}}=-\frac{1}{2} e^{T \bar{R}} e^{\mathrm{Q} \bar{S}} \partial_{[T} e_{\mathrm{Q}] \bar{T}} e_{R}^{\bar{T}}+\frac{1}{2} e^{S[\bar{R}} \partial_{[R} e_{S]}^{\bar{S}]},
$$

and $F(z)$ is some general scalar function, providing a mass term with a kink-like profile, which from this point is written in terms of a conformal variable $z$ such that $d z=e^{-A(y)} d y$ regards a transformation to conformal coordinates. This kind of mass term is introduced in the action, for it has played 


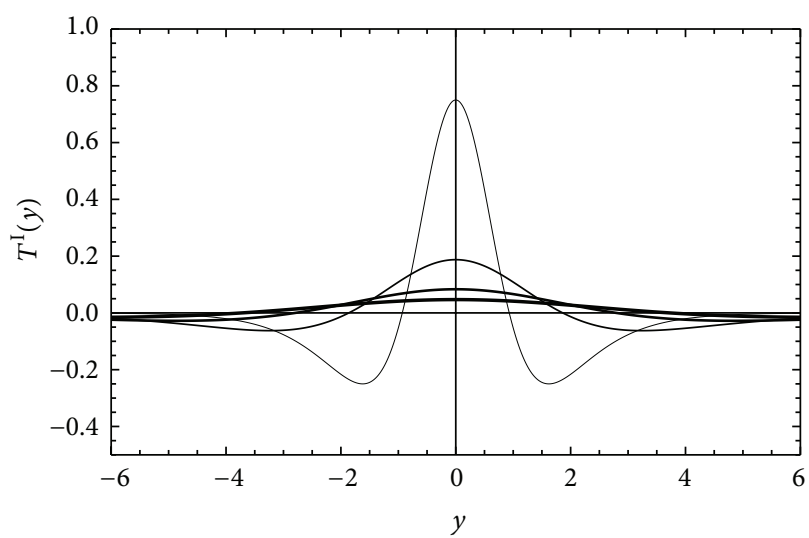

(a)

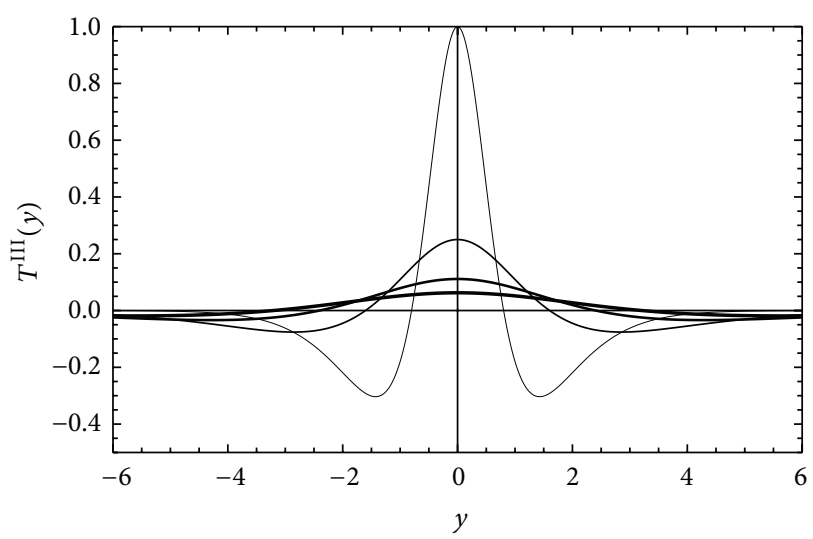

(c)

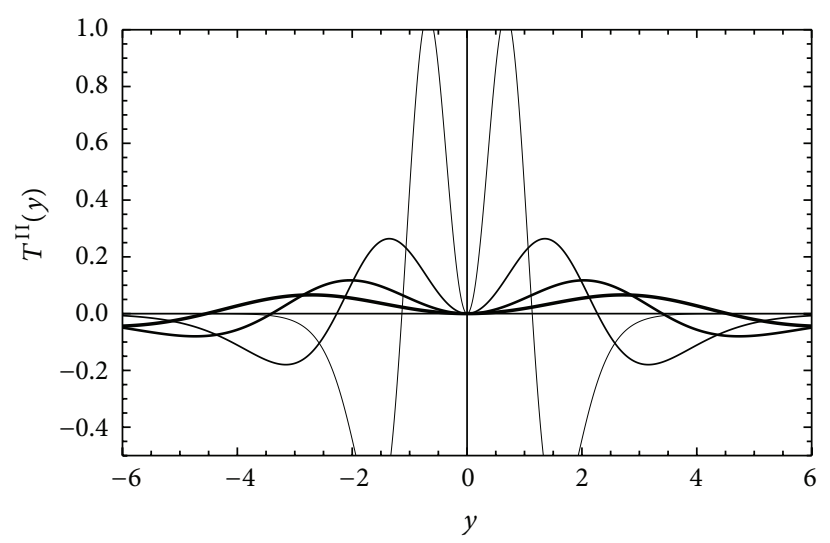

(b)

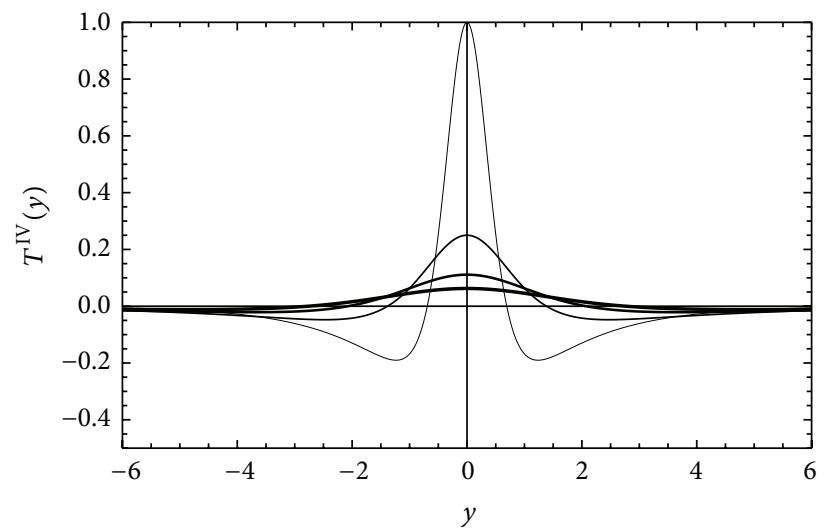

(d)

Figure 2: Energy density, $T_{00}(y)$, for models from I (a) to IV (d), where integer values of the brane width parameter $a$, running from 1 (thinnest line) to 4 (thickest line), corresponding to an increasing thickness.

a critical role on the localization of fermionic fields on a Minkowski brane. The components of the spin connection $\omega_{M}$ with respect to (1) are $\omega_{\mu}=(1 / 2)\left(\partial_{z} A\right) \gamma_{\mu} \gamma_{5}+\widehat{\omega}_{\mu}$, where $\widehat{\omega}_{\alpha}=(1 / 4) \bar{\omega}_{\alpha}^{\overline{\mu \nu}} \Gamma_{\bar{\mu}} \Gamma_{\bar{v}}$ is the spin connection derived from the metric $g_{\mu \nu}=\widetilde{e}_{\mu}^{\bar{\mu}} \widetilde{e}_{\nu}^{\bar{v}} \eta_{\overline{\mu \nu}}$. Thus, the equation of motion corresponding to the action (19) reads

$$
\left[\gamma^{\mu}\left(\partial_{\mu}+\widehat{\omega}_{\mu}\right)+\gamma^{5}\left(\partial_{z}+2 \partial_{z} A\right)-e^{A} M F(z)\right] \Psi=0
$$

The $5 D$ Dirac equation can be hence studied by taking spinors with respect to $4 D$ effective fields. In this way the chiral splitting yields

$$
\Psi=e^{-2 A(z)}\left(\sum_{n} \psi_{L n}\left(x^{\mu}\right) L_{n}(z)+\psi_{R n}\left(x^{\mu}\right) R_{n}(z)\right)
$$

where $L_{n}(z)$ and $R_{n}(z)$ are the well-known KK modes, and $\psi_{R n}\left(x^{\mu}\right)=+\gamma^{5} \psi_{R n}\left(x^{\mu}\right)\left[\psi_{L n}\left(x^{\mu}\right)=\gamma^{5} \psi_{L n}\left(x^{\mu}\right)\right]$ is the rightchiral [left-chiral] component of a $4 D$ Dirac field, respectively. In addition, the sum over $n$ can be both continuous and discrete. Assuming that $\gamma^{\mu}\left(\partial_{\mu}+\widehat{\omega}_{\mu}\right) \psi_{(R, L) n}=m_{n} \psi_{(L, R) n}$, the $L_{n}(z)$ and $R_{n}(z)$ functions should then satisfy the subsequent coupled equations,

$$
\begin{aligned}
& {\left[\partial_{z}-e^{A} M F(z)\right] R_{n}(z)=-m_{n} L_{n}(z),} \\
& {\left[\partial_{z}+e^{A} M F(z)\right] L_{n}(z)=m_{n} R_{n}(z) .}
\end{aligned}
$$

The associated Schrödinger-like equations can be thus acquired for the left- and right-chiral KK modes of fermions, respectively, as

$$
\begin{aligned}
& \left(-\partial_{z}^{2}+V_{L}(z)\right) L_{n}=m_{L_{n}}^{2} L_{n}, \\
& \left(-\partial_{z}^{2}+V_{R}(z)\right) R_{n}=m_{R_{n}}^{2} R_{n},
\end{aligned}
$$

where the mass-independent potentials are given by

$$
\begin{aligned}
V_{L}(z)= & e^{2 A} M^{2} F^{2}(z)-e^{A} A^{\prime} M F(z) \\
& -e^{A} M \partial_{z} F(z), \\
V_{R}(z)= & e^{2 A} M^{2} F^{2}(z)+e^{A} A^{\prime} M F(z) \\
& +e^{A} M \partial_{z} F(z) .
\end{aligned}
$$


Note that the Schrödinger-like equations (24) can be transformed into $U^{\dagger} U L_{n}=m_{n}^{2} L_{n}$ and $U U^{\dagger} R_{n}=m_{n}^{2} R_{n}$, where $U \equiv \partial_{z}+e^{A} M F(z)$. This observation is based upon supersymmetric quantum mechanics, implying that the mass squared is nonnegative.

In order to lead these results to the standard $4 D$ action for a massless fermion and a series of massive chiral fermions, the action $S=\sum_{n} \int d^{4} x \sqrt{-g} \bar{\psi}_{n}\left[\gamma^{\mu}\left(\partial_{\mu}+\widehat{\omega}_{\mu}\right)-m_{n}\right] \psi_{n}$ is employed, for orthonormalization conditions

$$
\begin{aligned}
& \int_{-\infty}^{+\infty} L_{m} L_{n} d z=\delta_{m n}=\int_{-\infty}^{+\infty} R_{m} R_{n} d z, \\
& \int_{-\infty}^{+\infty} L_{m} R_{n} d z=0 .
\end{aligned}
$$
yields

In formulae (23a) and (23b), by setting $m_{n}=0$, thus it

$$
\begin{aligned}
& L_{0} \propto e^{-M \int e^{A} F d z}, \\
& R_{0} \propto e^{M \int e^{A} F d z} .
\end{aligned}
$$

Hence, either the massless left- or right-chiral KK fermion modes can be localized on the brane, being the other one nonnormalizable.

By taking $F(z)=\zeta(z)$, regarding (16), it yields

$$
\begin{aligned}
& V_{L}(z(y))=e^{2 A(y)}\left(M^{2} \zeta^{2}(y)-\frac{d A(y)}{d y} M \zeta(y)\right. \\
& \left.-M \frac{d \zeta(y)}{d y}\right) \\
& V_{R}(z(y))=e^{2 A(y)}\left(M^{2} \zeta^{2}(y)+\frac{d A(y)}{d y} M \zeta(y)\right. \\
& \left.\quad+M \frac{d \zeta(y)}{d y}\right) .
\end{aligned}
$$

Equations (28a) and (28b) evince that when the mass term in the action (19) regards $M=0$, the potentials for leftand right-chiral KK modes $V_{L, R}(z)$ vanish. Then both chiral fermions cannot be localized on the thick brane. Moreover, if $V_{L}(z)$ and $V_{R}(z)$ are demanded to be $\mathbb{Z}_{2}$-even with respect to the extra dimension $z$, then the mass term $M F(z)$ must be an odd function of $z$ [29]. In fact, some useful classes of braneworld models have the extra dimension topology $S^{1} / \mathbb{Z}_{2}$. If the background scalar is an odd function of extra warped dimension, the Yukawa coupling, between the fermion and the background scalar field, assures the localization mechanism for fermions [29]. For the majority of brane-world models, the scalar field $\zeta$ is, usually, a kink, being an odd function of the extra dimension. Here we do not necessarily impose this condition, in order to not preclude asymmetric solutions, with respect to the extra dimension.

In what follows the profile of the above left-right potentials is depicted in Figure 3 for different values of the localization parameter $a$. In fact, the potentials $V_{L, R}(z)$ have asymptotic behaviors that tend to zero from up, as $y \rightarrow \pm \infty$, for all models from I to IV. In model I, at $y=0$ the potential $V_{R}(z)$ attains its maximum positive value, a global maximum, for $a=1$. The potential $V_{R}(z)$ changes to a volcano-type profile along the interval of $1<a<2$, such that for $a=2,3,4, \ldots$ the point $y=0$ regards a local minimum, which allows for producing unstable resonances, which can be tunneled to the outside of the potential. Nevertheless, the potential $V_{L}(z)$ has the associated minima at $y=0$ for all positive integer values of $a, a=2,3,4, \ldots$, and it creates the conditions for producing bound states. A very similar behavior is exhibited by model III, in spite of showing different amplitudes. Model IV is quite similar to these models, with the only qualitative difference concerning the fact that, at $y=0$, the potential $V_{R}(z)$ attains its maximum positive value, a global maximum, for $a=1$ and $a=2$. For model IV, the stability conditions created by the rightand left-chiral volcano-type potentials are more sensible to the increasing of the brane width $(a \gtrsim 3)$, in comparison to models I and II ones ( $a \gtrsim 2)$, inducing no mass gap to separate the fermion zero mode from the excited KK massive modes. In these cases, there exist continuous spectra for the KaluzaKlein modes of fermions of both chiralities. These volcanotype potentials imply the existence of resonant or metastable states of fermions which can tunnel from the brane to the bulk [9]. The left-chiral KK mode has a continuous gapless spectrum for models I, III, and IV, according to Figure 3. Since the potential for left-chiral fermions presents a negative value at the brane location for these models, the zero modes of right- and left-chiral fermions, $R_{0}(y)$ and $L_{0}(y)$, are the only necessary ingredient to be tested to be localized on the brane. For model II, both potentials for the left- and right-chiral fermions have positive values of the potential, irrespective of $y$. However for both cases $V_{R, L}(y)$, when $a \leqslant 1$, an asymmetric behavior emerges and produces a totally odd symmetric well-barrier profile in the limit of $a \rightarrow 0$. Except for $0<a \leqslant 1$, the zero mode of left- and right-chiral fermions can not be trapped. All potentials for model II are asymmetric (except for $a=0$, which is nonsense in the brane context), have maxima at $y=0$, and tend to zero at $y \rightarrow \pm \infty$, and there is no bound state for right-chiral fermions. In particular, for $V_{R, L}(y)$ when $a=1$, the minima occur at $y \sim \pm 0.87$.

\section{Matter Localization for Spin 0 Scalar Fields}

The localization of scalar fields on thick branes generated by deformed defects can also be considered from this point. In particular, an interesting approach on domain walls can be also found in [30]. In fact, a massive scalar field coupled to gravity can be described by the following action:

$$
S_{0}=-\frac{1}{2} \int d^{5} x \sqrt{-g}\left(g_{M N} \partial^{M} \Phi \partial^{N} \Phi+m_{0}^{2} \Phi^{2}\right),
$$

where $m_{0}$ denotes the effective mass of a bulk scalar field, $\Phi$, and from where one can check whether spin 0 matter fields can be trapped on the thick brane. By employing the metric 


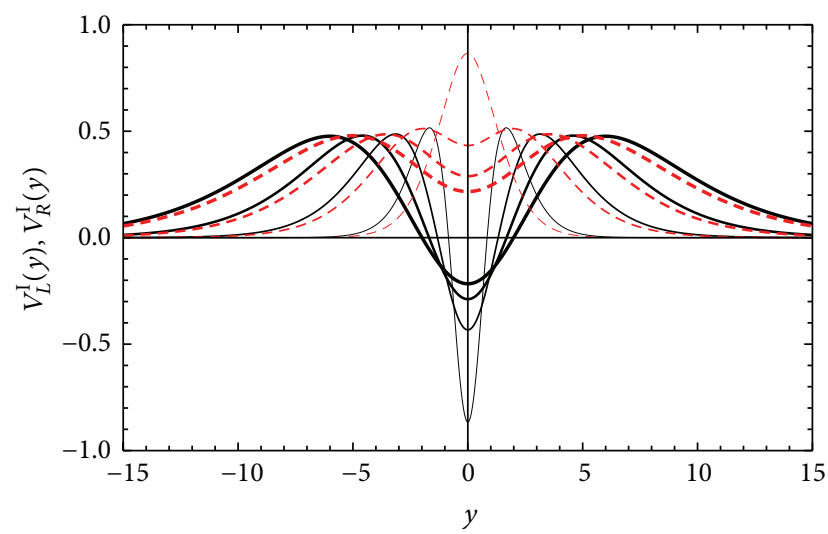

(a)

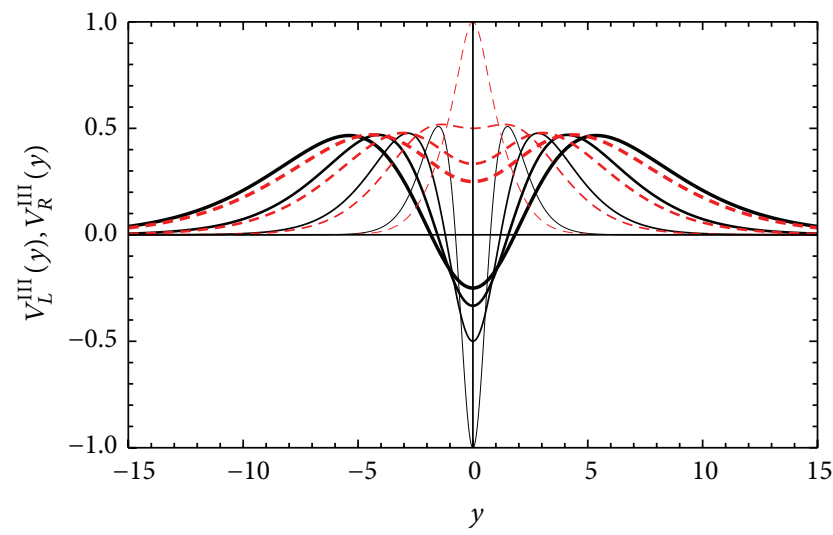

(c)

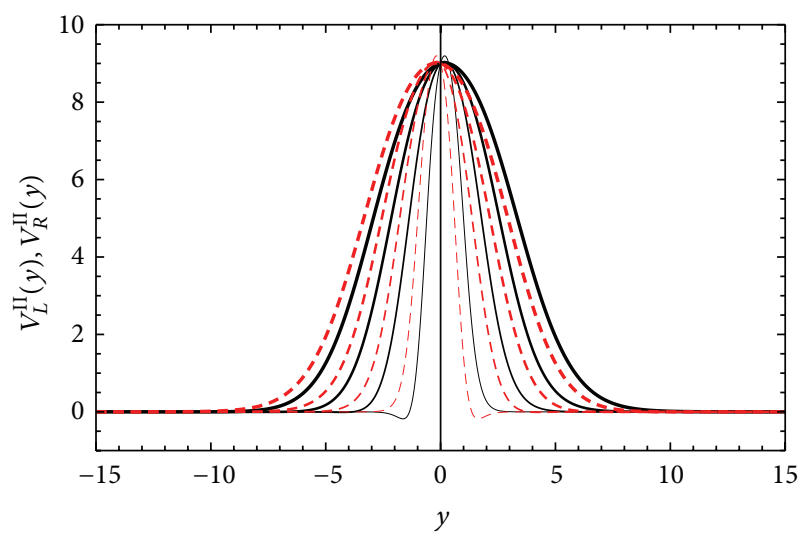

(b)

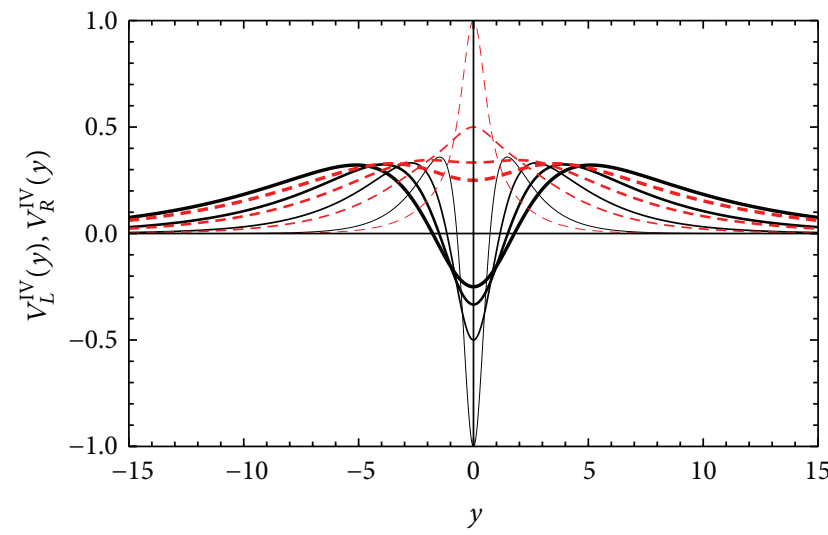

(d)

Figure 3: Associated Schrödinger-like quantum mechanical potentials, $V_{L}(y)$ and $V_{R}(y)$, respectively, for left-chiral (solid (black) lines) and right-chiral (dashed (red) lines) KK modes of fermions, for models from I (a) to IV (d). Again, one has considered integer values of the brane width parameter $a$, running from 1 (thinnest line) to 4 (thickest line), corresponding to an increasing thickness. The fermion mass parameter has been assumed to be equal to unit, $M=1$.

(1), the associated equation of motion from the action in (29) reads

$$
\begin{aligned}
& \frac{1}{\sqrt{-g}} \partial_{\mu}\left(\sqrt{-g} g^{\mu \nu} \partial_{\nu} \Phi\right)+e^{-3 A} \partial_{z}\left(e^{3 A} \partial_{z} \Phi\right)-e^{2 A} m_{0}^{2} \Phi \\
& \quad=0 .
\end{aligned}
$$

Hence, by the KK decomposition $\Phi\left(x^{\mu}, z\right)=$ $\sum_{n} \chi_{n}\left(x^{\mu}\right) \xi_{n}(z) e^{-3 A / 2}$, where $\xi_{n}$ is assumed to satisfy the $4 D$ Klein-Gordon equation $\left[\partial_{\mu}\left(\sqrt{-g} g^{\mu \nu} \partial_{\nu}\right) / \sqrt{-g}-m_{n}^{2}\right] \xi_{n}\left(x^{\mu}\right)=$ $0, m_{n}$ being the $4 D$ mass of the KK excitation of the scalar field. Then the scalar KK mode $\xi_{n}(z)$ is ruled by the following equation:

$$
\left[-\partial_{z}^{2}+V_{0}(z)\right] \xi_{n}(z)=m_{n}^{2} \xi_{n}(z)
$$

This equation is a Schrödinger one, with effective potential given by

$$
\begin{aligned}
V_{0} & (z(y))=\frac{3}{2} A^{\prime \prime}(z)+\frac{9}{4} A^{\prime 2}(z)+e^{2 A(z)} m_{0}^{2} \\
= & e^{2 A(z)}\left(\frac{3}{2} \frac{d^{2} A(y)}{d y^{2}}+\frac{15}{4}\left(\frac{d A(y)}{d y}\right)^{2}+m_{0}^{2}\right) .
\end{aligned}
$$

The profile of the above scalar boson potential is depicted in Figure 4 (solid (black) lines) for different values of the localization parameter $a$. For $m_{0}=1$, only brane scenarios with $a \lesssim 2$ provide conditions to have a localized scalar field. Even in this case, such localized states behave much more as resonances than as bound states, given that they can be tunneled out of the potential. Bound states appear only for noninteger values of the brane width such that $a<1$, which will correspond to typical volcano-type potentials.

\section{Matter Localization for Spin 1 Vector Fields}

One now turns to spin 1 vector fields and begins with the $5 D$ action of a vector field:

$$
S_{1}=-\frac{1}{4} \int d^{5} x \sqrt{-g} g^{M N} g^{R S} F_{M R} F_{N S}
$$

where $F_{M N}=\partial\left[{ }_{M} A_{N}\right]$ denotes the field strength tensor. A $5 D$ spin 1 field can be now studied via the KK decomposition $A_{M}\left(x^{\rho}, z\right)=\sum_{n} a_{M}^{(n)}\left(x^{\rho}\right) \tau_{n}(z)$. The action of the $5 D$ massless 


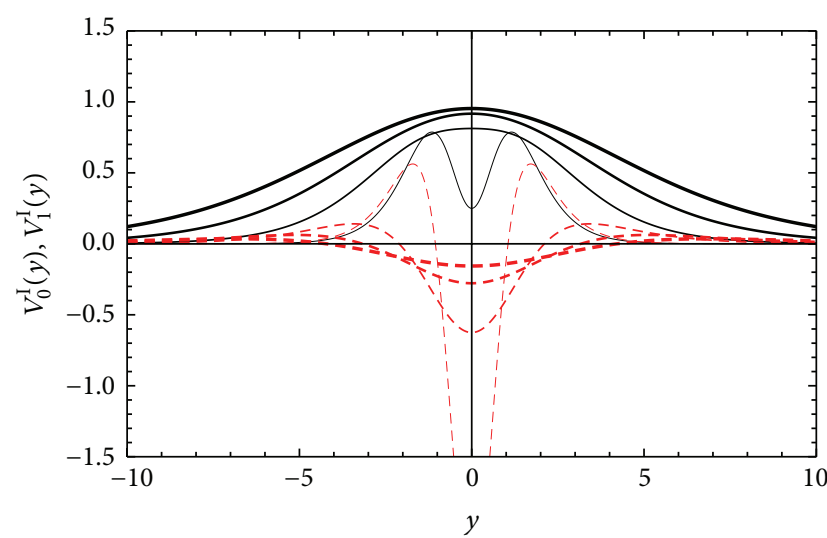

(a)

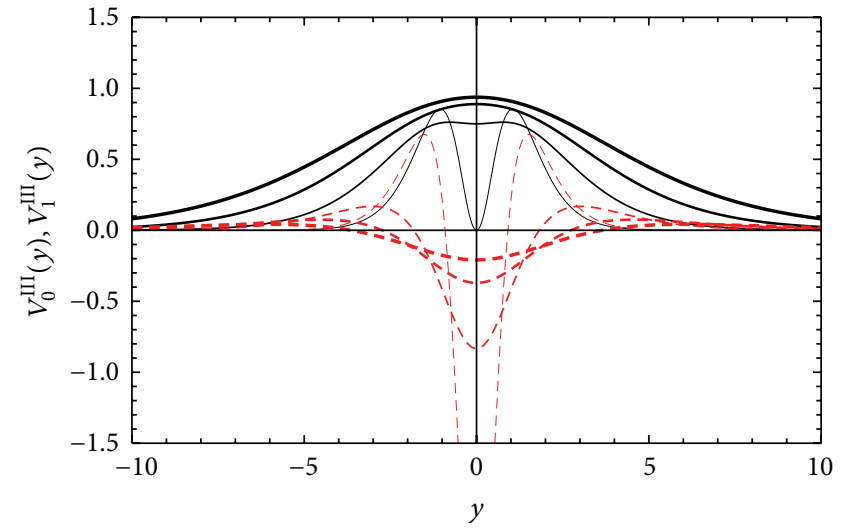

(c)

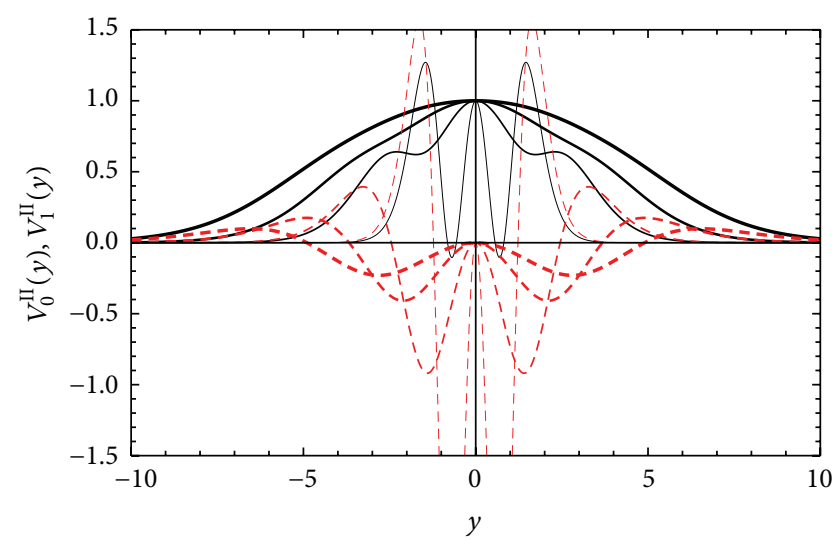

(b)

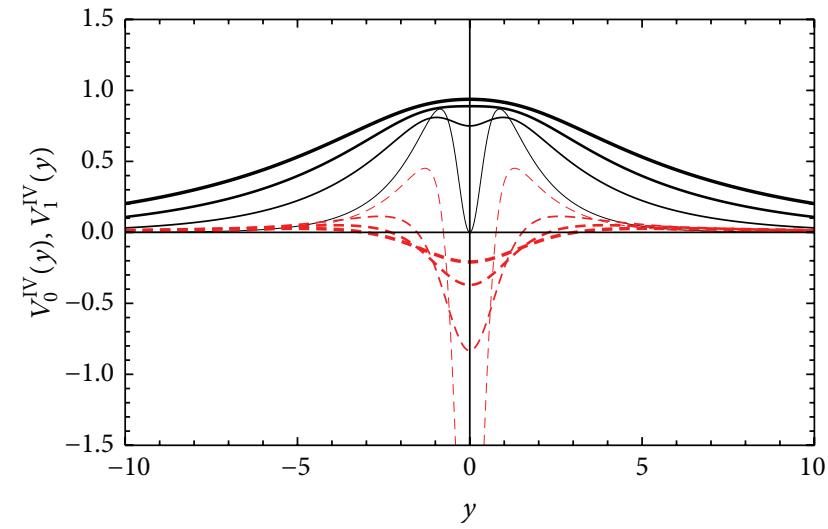

(d)

FIGURE 4: Associated Schrödinger-like quantum mechanical potentials, $V_{0}(y)$ and $V_{0}(y)$, respectively, for spin 0 (solid (black) lines) and spin 1 (dashed (red) lines) bosons for models from I (a) to IV (d). Again, one has considered integer values of the brane width parameter $a$ running from 1 (thinnest line) to 4 (thickest line), corresponding to an increasing thickness. The spin 0 boson mass parameter has been assumed to be equal to unit.

vector field (33) is invariant under the following gauge transformation:

$$
\begin{aligned}
A_{M}\left(x^{\rho}, z\right) & \longmapsto \widetilde{A}_{M}\left(x^{\rho}, z\right) \\
& =A_{M}\left(x^{\rho}, z\right)+\partial_{M} F\left(x^{\rho}, z\right),
\end{aligned}
$$

where $F\left(x^{\rho}, z\right)$ denotes any arbitrary regular scalar function, for $M=\mu, 5$. The field component $A_{5}\left(x^{\rho}, z\right)$ equals zero [25], by this gauge. In fact, (34) yields

$$
\widetilde{A}_{5}\left(x^{\rho}, z\right)=\sum_{n} a_{5}^{(n)}\left(x^{\rho}\right) \tau_{n}(z)+\partial_{z} F\left(x^{\rho}, z\right)
$$

By choosing $F\left(x^{\rho}, z\right)=-\sum_{n} a_{5}^{(n)}\left(x^{\rho}\right) \int \tau_{n}(z) d z,[25]$ then $\widetilde{A}_{5}=0$, and hence the action (33) leads to the space-time action

$$
S_{1}=-\frac{1}{4} \int d^{5} x \sqrt{-g}\left(F^{\mu \nu} F_{\mu \nu}+2 e^{-A} g^{\mu \nu} A_{\mu}^{\prime} A_{\nu}^{\prime}\right)
$$

where ()$^{\prime}=\partial_{z}$. Given a set of orthonormal functions $\tau_{n}(z)$, playing the role of spin 1 Kaluza-Klein modes, and the decomposition of the vector field $A_{\mu}\left(x^{\rho}, z\right)=\sum_{n} a_{\mu}^{(n)}\left(x^{\mu}\right) \tau_{n}(z) e^{-A / 2}$, the action (36) reads

$$
S_{1}=-\frac{1}{2} \sum_{n} \int d^{4} x \sqrt{-g}\left(\frac{1}{2} f^{(n)^{\mu \nu}} f_{\mu \nu}^{(n)}+m_{n}^{2} a^{(n)^{\mu}} a_{\mu}^{(n)}\right),
$$

where $f_{\mu \nu}^{(n)}=\partial_{[\mu} a_{\nu]}^{(n)}$ stands for the $4 D$ field strength tensor. The KK modes $\tau_{n}(z)$ satisfy the Schrödinger equation

$$
\left(-\partial_{z}^{2}+V_{1}(z)\right) \tau_{n}(z)=m_{n}^{2} \tau_{n}(z),
$$

where the mass-independent potential reads [31]

$$
\begin{aligned}
V_{1}(z) & =\frac{1}{4} A^{\prime 2}(z)+\frac{1}{2} A^{\prime \prime}(z) \\
& =e^{2 A(z)}\left[\frac{1}{2} \frac{d^{2} A(y)}{d y^{2}}+\frac{3}{4}\left(\frac{d A(y)}{d y}\right)^{2}\right] .
\end{aligned}
$$

The profile of the above vector boson potential is depicted in Figure 4 (dashed (red) lines) for different values of 
the localization parameter $a$. All the thick brane scenarios with $a \gtrsim 1$ provide localization conditions to have vector field bound states. Increasing values of $a$ lead to more stable bound states.

\section{Conclusions and Discussion}

Thick branes driven by superpotentials supported by deformed defects (c.f. (13)-(15)) for various bulk matter fields of spin 0 , spin $1 / 2$, and spin 1 have been investigated. For spin 1 gauge fields, the profile of the associated vector boson potential showed that, in the thick brane models for $a \geqslant 1$, localization conditions hold as to guarantee the existence of vector field bound states. Quantitatively, increasing values of the brane thickness parameter, $a$, lead to more stable bound states.

Concerning spin 0 (scalar) fields, the profile of the potential evinces that only thick brane scenarios with $a \gtrsim$ 4 provide localization conditions compatible to scalar field bound states.

The most intricate result is related to spin $1 / 2$ fields. In fact, for fermionic fields, left-right potentials were deeply studied and for the four models considered here, the issue of localization has been scrutinized. It is worth pointing out that models I, III, and IV admit volcano-type potentials, inducing no mass gap to separate the fermion zero mode from the excited KK massive modes. Hence continuous spectra for the Kaluza-Klein modes of fermions of both chiralities are allowed. A refined analysis of the values of the $a$ parameter in these models, influencing the localization of fermionic fields, was provided. Model II, induced by the superpotential (14), reveals a peculiar behavior. In this model, right-chiral fermions have positive values of the potential irrespective of the extra dimension when $a=1$. Hence, except for this value, the zero mode of left- and right-chiral fermions can not be trapped. All potentials for model II are asymmetric and have maxima at $y=0$ and minima at $y \rightarrow \pm \infty$, and there is no bound state for right-chiral fermions, but, again, for $V_{R, L}(y)$ when $a=1$.

It is worth mentioning that, for the localization of a fermion zero mode, the mass term $M F(z) \bar{\Psi} \Psi$ was considered in the $5 D$ action. An interesting approach concerning such mass term in (19) has been studied, corresponding to the so-called singular dark spinors $[32,33]$. Such massive mass dimension one quantum fields are prime candidates for the dark matter problem, also presenting possible signatures at LHC [34]. It generates a slightly different action responsible for spin 1/2 matter fields localization $[35,36]$. Such approach can be also extended in the context of deformed defects here presented.

\section{Competing Interests}

The authors declare that they have no competing interests.

\section{Acknowledgments}

The work of Alex E. Bernardini is supported by the Brazilian Agencies FAPESP (Grant 2015/05903-4) and CNPq (Grant no. 300809/2013-1 and Grant no. 440446/2014-7). Roldão da Rocha is grateful to CNPq (Grants no. 303293/2015-2 and no. 473326/2013-2) and to FAPESP (Grant 2015/10270-0) for partial financial support.

\section{References}

[1] I. Antoniadis, N. Arkani-Hamed, S. Dimopoulos, and G. Dvali, "New dimensions at a millimeter to a fermi and superstrings at a TeV," Physics Letters B, vol. 436, no. 3-4, pp. 257-263, 1998.

[2] L. Randall and R. Sundrum, "Large mass hierarchy from a small extra dimension," Physical Review Letters, vol. 83, no. 17, pp. 3370-3373, 1999.

[3] O. DeWolfe, D. Z. Freedman, S. S. Gubser, and A. Karch, "Modeling the fifth dimension with scalars and gravity," Physical Review D, vol. 62, no. 4, Article ID 046008, 2000.

[4] M. Gremm, "Four-dimensional gravity on a thick domain wall," Physics Letters B, vol. 478, no. 4, pp. 434-438, 2000.

[5] D. Bazeia, C. Furtado, and A. R. Gomes, "Brane structure from a scalar field in warped spacetime," Journal of Cosmology and Astroparticle Physics, vol. 2004, no. 2, article 002, 2004.

[6] X.-H. Zhang, Y.-X. Liu, and Y.-S. Duan, "Localization of fermionic fields on braneworlds with bulk tachyon matter," Modern Physics Letters A, vol. 23, no. 25, pp. 2093-2101, 2008.

[7] A. A. Andrianov, V. A. Andrianov, and O. O. Novikov, "Gravity effects on thick brane formation from scalar field dynamics," European Physical Journal C, vol. 73, article 2675, 2013.

[8] Y.-X. Liu, C.-E. Fu, L. Zhao, and Y.-S. Duan, "Localization and mass spectra of fermions on symmetric and asymmetric thick branes," Physical Review D, vol. 80, no. 6, Article ID 065020, 2009.

[9] Z.-H. Zhao, Y.-X. Liu, H.-T. Li, and Y.-Q. Wang, "Effects of the variation of mass on fermion localization and resonances on thick branes," Physical Review D, vol. 82, no. 8, Article ID 084030, 10 pages, 2010.

[10] V. Dzhunushaliev, V. Folomeev, and M. Minamitsuji, “Thick brane solutions," Reports on Progress in Physics, vol. 73, no. 6, Article ID 066901, 2010.

[11] D. Bazeia, L. Losano, and J. M. C. Malbouisson, "Deformed defects," Physical Review D, vol. 66, no. 10, Article ID 101701, 2002.

[12] A. E. Bernardini and R. da Rocha, "Cyclically deformed defects and topological mass constraints," Advances in High Energy Physics, vol. 2013, Article ID 304980, 18 pages, 2013.

[13] R. A. Correa, A. de Souza Dutra, and M. B. Hott, "Fermion localization on degenerate and critical branes," Classical and Quantum Gravity, vol. 28, no. 15, Article ID 155012, 2011.

[14] G. German, A. Herrera-Aguilar, D. Malagon-Morejon, R. R. Mora-Luna, and R. da Rocha, "A de Sitter tachyon thick braneworld and gravity localization," Journal of Cosmology and Astroparticle Physics, vol. 1302, p. 035, 2013.

[15] G. Germán, A. Herrera-Aguilar, D. Malagón-Morejón, I. Quiros, and R. da Rocha, "Study of field fluctuations and their localization in a thick braneworld generated by gravity nonminimally coupled to a scalar field with the Gauss-Bonnet term," Physical Review D, vol. 89, no. 2, Article ID 026004, 2014.

[16] A. E. Bernardini, R. T. Cavalcanti, and R. da Rocha, "Spherically symmetric thick branes cosmological evolution," General Relativity and Gravitation, vol. 47, article 1840, 2015. 
[17] A. Ahmed and B. Grzadkowski, "Brane modeling in wraped extra-dimension," Journal of High Energy Physics, vol. 2013, article 177, 2013.

[18] N. Barbosa-Cendejas, D. Malagón-Morejón, and R. R. MoraLuna, "Universal spin-1/2 fermion field localization on a 5D braneworld," General Relativity and Gravitation, vol. 47, article 77, 2015.

[19] W. T. Cruz, R. V. Maluf, and C. A. S. Almeida, "Kalb-Ramond field localization on the Bloch brane," The European Physical Journal C, vol. 73, p. 2523, 2013.

[20] I. Oda, "Locally localized gravity models in higher dimensions," Physical Review D, vol. 64, no. 2, Article ID 026002, 11 pages, 2001.

[21] C. A. Almeida, R. Casana, J. Ferreira, and A. R. Gomes, "Fermion localization and resonances on two-field thick branes," Physical Review D. Particles, Fields, Gravitation, and Cosmology, vol. 79, no. 12, 125022, 12 pages, 2009.

[22] D. Bazeia, R. Menezes, and R. da Rocha, "A note on asymmetric thick branes," Advances in High Energy Physics, vol. 2014, Article ID 276729, 8 pages, 2014.

[23] D. Bazeia, L. Losano, R. Menezes, and R. da Rocha, "Study of models of the sine-Gordon type in flat and curved spacetime," European Physical Journal C, vol. 73, article 2499, 2013.

[24] A. de Souza Dutra, G. P. de Brito, and J. M. Hoff da Silva, "Asymmetrical Bloch branes and the hierarchy problem," EPL (Europhysics Letters), vol. 108, no. 1, p. 11001, 2014.

[25] H. Guo, A. Herrera-Aguilar, Y.-X. Liu, D. Malagón-Morejón, and R. R. Mora-Luna, "Localization of bulk matter fields, the hierarchy problem and corrections to Coulomb's law on a pure de Sitter thick braneworld," Physical Review D, vol. 87, no. 9, Article ID 095011, 21 pages, 2013.

[26] A. Herrera-Aguilar, D. Malagón-Morejón, and R. R. MoraLuna, "Localization of gravity on a de Sitter thick braneworld without scalar fields," Journal of High Energy Physics, vol. 2010, article 15, 2010.

[27] R. Maartens and K. Koyama, "Brane-world gravity," Living Reviews in Relativity, vol. 13, article 5, 2010.

[28] A. E. Bernardini and O. Bertolami, "Equivalence between BornInfeld tachyon and effective real scalar field theories for brane structures in warped geometry," Physics Letters B, vol. 726, no. 1-3, pp. 512-517, 2013.

[29] Y.-X. Liu, Z.-G. Xu, F.-W. Chen, and S.-W. Wei, "New localization mechanism of fermions on braneworlds," Physical Review D, vol. 89, no. 8, Article ID 086001, 5 pages, 2014.

[30] D. Stojkovic, "Fermionic zero modes on domain walls," Physical Review D, vol. 63, no. 2, Article ID 025010, 2000.

[31] Q.-Y. Xie, Z.-H. Zhao, Y. Zhong, J. Yang, and X.-N. Zhou, "Localization and mass spectra of various matter fields on scalar-tensor brane," Journal of Cosmology and Astroparticle Physics, vol. 2015, no. 3, article 014, 2015.

[32] D. V. Ahluwalia, C. Y. Lee, and D. Schritt, "Self-interacting Elko dark matter with an axis of locality," Physical Review D, vol. 83, no. 6, Article ID 065017, 2011.

[33] R. da Rocha, A. E. Bernardini, and J. M. Hoff da Silva, "Exotic dark spinor fields," Journal of High Energy Physics, vol. 2011, article 110, 2011.

[34] M. Dias, F. de Campos, and J. M. Hoff da Silva, "Exploring Elko typical signature," Physics Letters B, vol. 706, no. 4-5, pp. 352359, 2012.

[35] I. C. Jardim, G. Alencar, R. R. Landim, and R. N. Costa Filho, "Solutions to the problem of Elko spinor localization in brane models," Physical Review D. Particles, Fields, Gravitation, and Cosmology, vol. 91, no. 8, Article ID 085008, 6 pages, 2015.

[36] Y. X. Liu, X. N. Zhou, K. Yang, and F. W. Chen, "Localization of 5D Elko spinors on Minkowski branes," Physical Review D, vol. 86, Article ID 064012, 2012. 

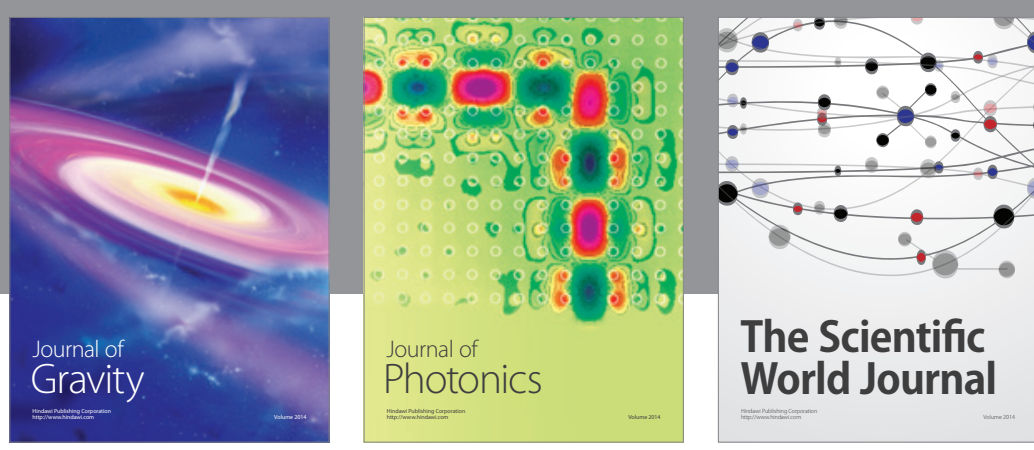

The Scientific World Journal
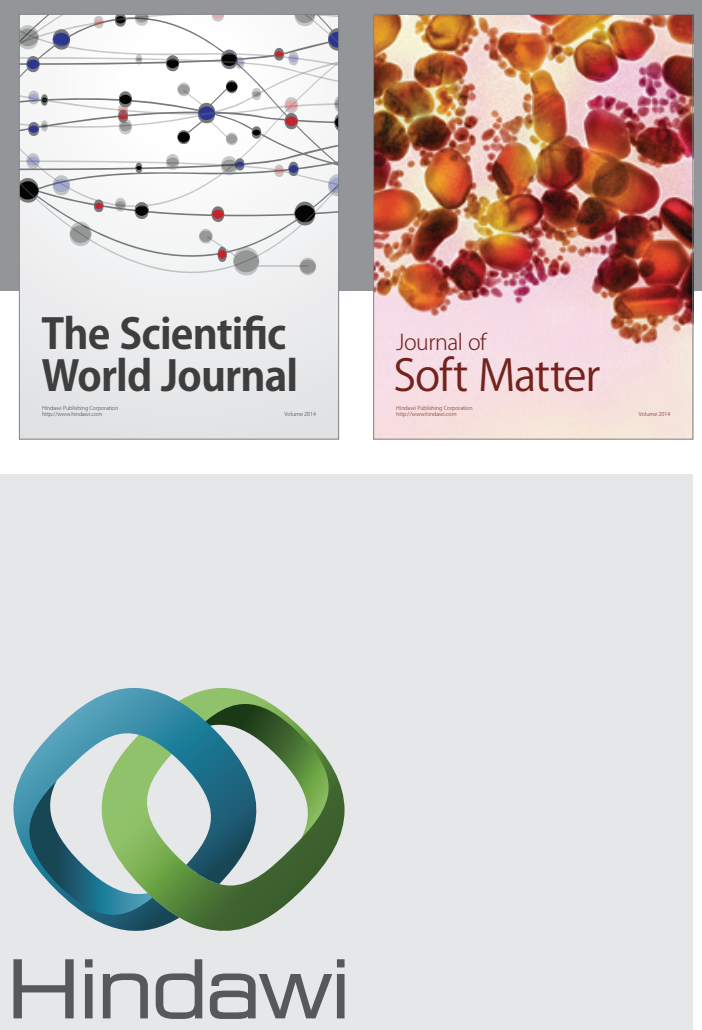

Submit your manuscripts at

http://www.hindawi.com

nternational Journal of

Statistical Mechanics
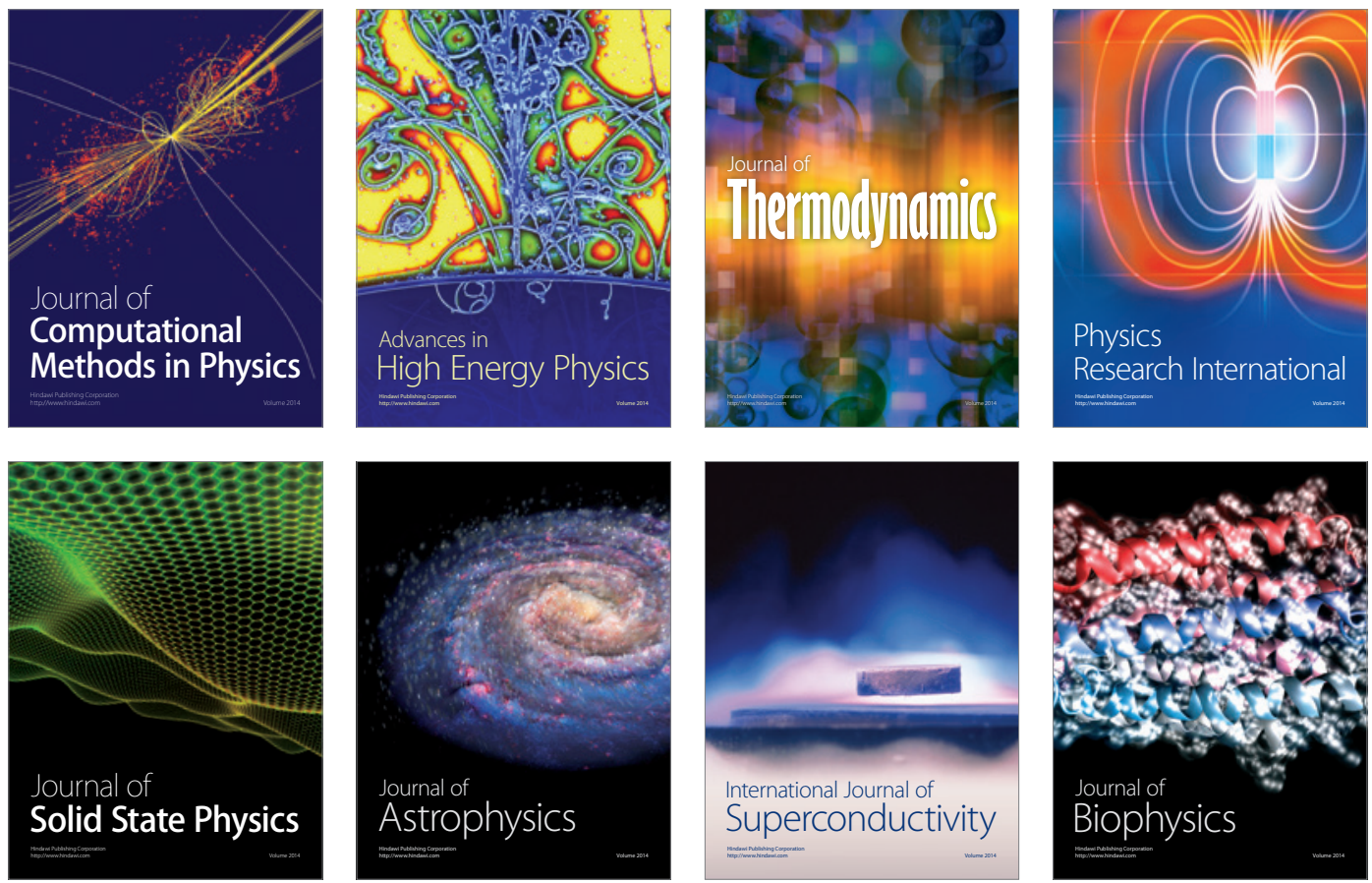
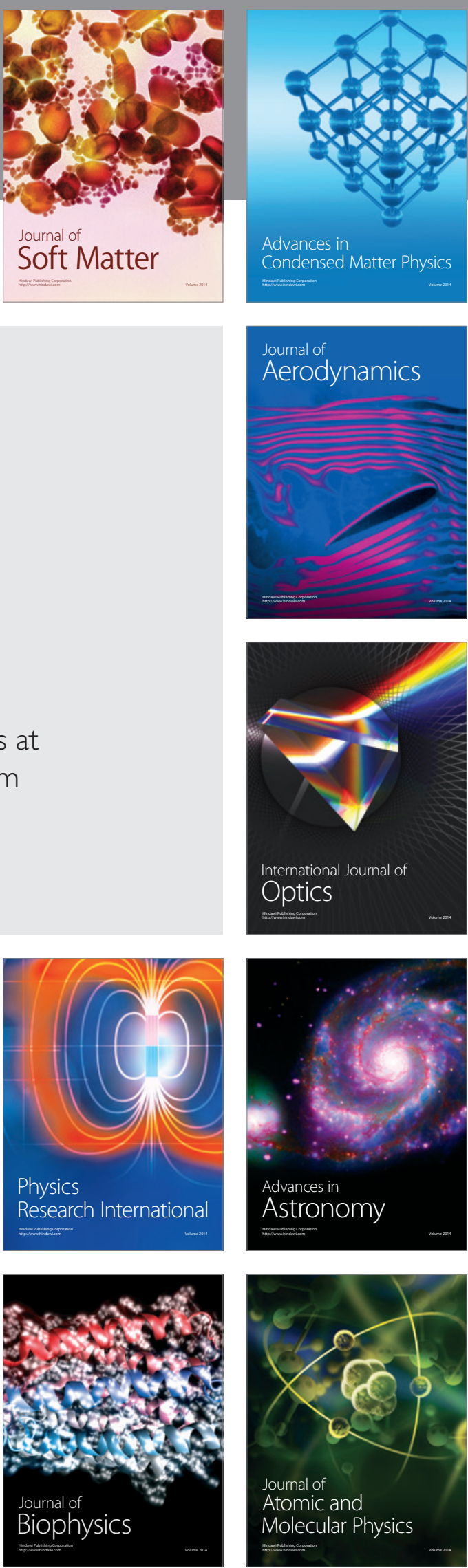